\title{
Paradigmas de la mejora y el control de la gestión pública (transparencia, responsabilidad y calidad de los servicios): perspectivas desde el ordenamiento jurídico español
}

\author{
Pedro T. Nevado-Batalla Moreno \\ Doctor en Derecho por la Universidad de Salamanca, España; licenciado en Derecho por la Universidad \\ de Salamanca; profesor titular de Derecho Administrativo de la Universidad de Salamanca, ex consejero de \\ Administración Pública Comunidad Autónoma de Extremadura, presidente del Consejo Consultivo de Extremadura. \\ Correo electrónico: pnevado@usal.es
}

\begin{abstract}
Resumen
En el presente texto se reflexiona sobre la gestión pública, a partir de la sencillez del objetivo y dificultad para su alcance. La transparencia: desde el conocimiento a la regeneración democrática, así como la responsabilidad: desde el olvido a la ejemplaridad, desde responsabilidad y control de nuevas perspectivas, finalmente, sobre la calidad de los servicios: desde la idea inalcanzable a la realidad mediante la evaluación. Palabras clave: Transparencia, buen gobierno, democracia, responsabilidad, calidad, servicios públicos.
\end{abstract}

\begin{abstract}
In this text, we reflection governance form simplicity of purpose to the afficulty to reach. Trasnparency: from the perceptive of knowledge to the democratic regeneration. Responsability from its oblivion to the exemplary and from responsability, and control of new perspective. finally the text exposes qualitie of services: form a unatanable idea to it reality trough evaluator.
\end{abstract}

Keywords: Governance, transparency, democracy, responsability, qualitie of services.

\section{Résumé}

Dans ce texte, nous réfléchissons sur la gouvernance, de la simplicité de l'objet et de la difficulté d'accès. Transparence: de la connaissance à la régénération et la responsabilité démocratique: de l'oubli à l' exemplaire, de la responsabilité et le contrôle des nouvelles perspectives en fin de compte la qualité des services: de l'idée à la réalité inaccessible par l'évaluation.

Mots-clés: la transparence, la bonne gouvernance, la démocratie, la responsabilité, les services publics de qualité. 



\section{Paradigmas de la mejora y el control de la gestión pública (transparencia, responsabilidad y calidad de los servicios): perspectivas desde el ordenamiento jurídico español ${ }^{*}$}

Pedro T. Nevado-Batalla Moreno

\section{INTRODUCCIÓN}

\section{La mejora de la gestión pública: sencillez del objetivo y dificultad para su alcance}

Aproximarse a las ideas de mejora y control de la gestión pública es aproximarse a un volumen poco abarcable de normas, estudios, programas y proyectos, que están repartidos a todos los niveles imaginables: gubernamentales, no gubernamentales, académicos, públicos, privados. Es más, tal es la preocupación e interés por esta materia (como no podía ser de otra manera, como ya hemos dicho en varias ocasiones y volveremos a reiterar, la mejor o peor gestión pública se vincula, en síntesis, al buen rendimiento de los pilares sobre los que se asienta cualquier Estado democrático y de derecho) que se ha llegado a generar incluso un nada desdeñable nicho de negocio en el ámbito de la consultoría y asistencia privada, que aportan en muchas ocasiones lo que se ha olvidado que se dispone o directamente no se quiere hacer en el sector público ${ }^{1}$.

El texto se deriva del trabajo sobre transparencia en la gestión pública.

1 Y decimos bien, "no se quiere hacer" ya que, hablando de administración pública debe traerse a colación esa vieja regla general, habitualmente positivizada en los diferentes bloques jurídicoadministrativo, sobre el carácter irrenunciable de la competencia que, conjugada con el deber de realizar las actividades materialmente posibles, nos perfilan un ámbito objetivo en el que el encaje de la actividad de consultoría y asistencia externa resulta muy estrecho cuando no es evidencia de una incapacidad que, en puridad jurídica, resulta inadmisible. 
Conceptos y planteamientos como reforma, reordenación, modernización, y de manera más depurada, buen gobierno o buena administración conforman el obligado contexto en el que en la actualidad se mueve la administración pública o, de manera más general, el sector público, al convertirse en el objetivo de cualquier gestor público, en especial de la burocracia política, sobre todo cuando en un contexto de profunda crisis económica y política (podríamos decir también que administrativa) el sector público, la administración pública, forman parte de la etiología del problema, incluso, para algunos no es simplemente una causa, si no el problema en sí.

Es evidente que no podemos estar al lado de este tipo de posturas, puesto que quien debe ser solución y garantía de un Estado que permite y posibilita el desarrollo de los derechos y el cumplimiento de las obligaciones propias de un sistema democrático, social y de derecho, no puede ser un obstáculo en perversa e inaceptable mutación. Pues bien, podemos convenir que, en general, el objetivo es diáfano y a estas alturas nombrado y reiterado en demasía, si es que, lo que es intrínsecamente bueno para un sistema, a fuerza de repetirlo someramente por no obtener los resultados adecuados, puede llegar a fatigar hasta alcanzar un peligroso nivel de desinterés social. La mejora de la administración para una óptima prestación de servicios de calidad, como idea síntesis, refleja, al fin y al cabo, que los receptores de dichos servicios, los ciudadanos, ven respondidas de manera adecuada sus necesidades y expectativas y, por tanto, perciben de manera tangible que su confianza no se ve defraudada más allá de las palabras o del contenido de textos normativos.

Este planteamiento que ostenta cronológicamente la actualidad del momento en el que se realiza carece en realidad de cualquier nota que pudiera calificarlo como contemporáneo, dado que la buena gestión de los asuntos públicos, el buen gobierno, en definitiva, ha sido una necesidad constante en la conformación política de las sociedades y su paso a la constitución de Estados sostenidos por una administración pública como integrante del Poder Ejecutivo. Desde este enfoque, bien se podrían escribir varias y muy extensas monografías sobre la cuestión apuntada que completarían las ya elaboradas, pero a título meramente ejemplificativo y sin ánimo de exhaustividad (también como recuerdo al bicentenario que se celebró hace un año) baste recordar diversos textos vinculados al origen del constitucionalismo hispanoamericano. Por ejemplo, el artículo 54 de la Constitución de Cundinamarca de 1811, alude:

La primera obligación del Poder Ejecutivo es y será siempre poner en ejecución y dar cumplimiento en todas sus partes a esta Constitución, impidiendo que el trascurso del tiempo y descuido introduzcan abusos y corruptelas contrarias a lo que en ella se dispone.

Por su parte, el artículo 13 de la Constitución de Cádiz de 1812 expone: "El objeto del Gobierno es la felicidad de la Nación, puesto que el fin de toda sociedad política no es otro que el bienestar de los individuos que la componen". 
El artículo 24 de la Constitución de Apatzingán de 1814 dicta:

La felicidad del pueblo y de cada uno de los ciudadanos consiste en el goce de la igualdad, seguridad, propiedad y libertad. La íntegra conservación de estos derechos es el objeto de la institución de los gobiernos, y el único fin de las asociaciones políticas.

Y el artículo 10 de la Constitución del Salvador de 1824 dictamina: "El Gobierno del Estado, es popular representativo; y la felicidad de este en la Federación, es su principal objeto".

A todas luces pueden evidenciarse dos ideas. Por un lado, expresado de manera muy sencilla y gráfica, que el fin de la administración no es otro que la felicidad de los ciudadanos. Afirmación que, entendida de manera amplia y desde una perspectiva jurídica, supone un estado de legalidad óptimo a través del cumplimiento de los objetivos normativamente establecidos que conforman una organización pública servicial y satisfactoria que responde de manera adecuada a las necesidades y expectativas de los ciudadanos.

Pero aún hay más, este objetivo de felicidad ciudadana que marca el norte que debe guiar toda actuación pública, ha supuesto y supone un punto cardinal que no debe perderse y, de hacerlo, debe retomarse so pena de caer en una grave desorientación que afecta al conjunto de la sociedad y a la propia estructura institucional de esta representada, para nosotros, por la administración pública.

Por otro lado, subyace la idea de control, de responsabilidad, siendo especialmente atinada la expresión del citado artículo 54 de la Constitución de Cundinamarca de 1811, al afirmar la obligación de impedir que el trascurso del tiempo y descuido introduzcan abusos y corruptelas contrarias a lo establecido en la Constitución, y por tanto, en el resto del ordenamiento jurídico. Trascurso del tiempo y descuido o lo que es, tal vez, lo mismo, confianza, acomodación y, en todo caso, falta de diligencia. Circunstancias que de manera crecida podrían proporcionar una explicación a la coyuntura político-administración que de manera globalizada se nos muestra con la crudeza de lamentables malas prácticas administrativas y escandalosos casos de corrupción pública, en su conjunto todos ellos reprochables y síntomas de una nociva patología que, creemos y así lo expondremos más adelante, va más allá de lo que el derecho y, en especial, el derecho administrativo puede aportar.

En síntesis, la idea de felicidad de los ciudadanos como objetivo de la administración, o con una semántica más actual, el concepto de buena administración en la gestión de los asuntos públicos, resulta fundamental desde el hito cronológico que nosotros hemos tomado en la Constitución de Cádiz dos veces centenaria. Y esta mediata formalización no es proporcional al desarrollo obtenido si tomamos como patrón el nivel de satisfacción de aquellos a quienes se les debe proporcionar felicidad o los grados de incumplimientos medidos fácticamente en número y gravedad a través de los casos de malas prácticas y corrupción conocidos. Por tanto, siendo un objetivo sencillo, intuitivo incluso para cualquier persona, su consecución no parece resultar en absoluto sencilla. 
No sería equilibrado el razonamiento expuesto si no se reconocieran los avances y prácticas reconocidas como positivas o de buena administración, pero incluso desde este reconocimiento, la desproporción sigue manteniéndose, lastrando peligrosamente la confianza y legitimidad de la administración frente a ciudadanos infelices por insatisfacción y la observación de fatales ejemplos de mala administración. Y es que, como acertadamente expone Habermas, un modelo u ordenamiento social que aspire a perdurar, debe contar con el consentimiento necesario de los ciudadanos más allá de la mera legitimación por la autoridad.

\section{CAMBIO Y MEJORA DE LA ADMINISTRACIÓN}

\section{Consideraciones generales}

Sin entrar a conocer todos los elementos que, con seguridad, podrían conformar una auténtica teoría general sobre la reforma y cambio de la administración, sí conviene hacer varias reflexiones sobre la cuestión. Lo anterior, con el objeto de trazar unos perfiles delimitadores del campo en el que los paradigmas de la mejora y control de la gestión pública han de ubicarse; serían cuatro las consideraciones por apuntar.

\section{Utilidad de la administración}

En primer lugar, por obvio que pueda resultar, confirmar la utilidad de la administración pública; su existencia se confirma tanto por su reconocimiento institucional en los correspondientes textos fundamentales, como por la actividad que desarrolla aun siendo conscientes de las máculas y deficiencias que puedan darse. Esto se reitera debido a que la realidad social muestra manifestaciones muy críticas respecto a la administración y su burocracia (tanto técnica como política) que no se corresponde con ese reconocimiento y el propio valor de la actividad desarrollada. Se trata de un continuo estado de opinión que se ha visto incrementado por la situación de anormalidad y gravedad económica que actualmente se padece y que se ha inoculado de manera alarmante en la sociedad, hasta el punto de resultar un problema reconducir dicha opinión a los parámetros de racionalidad que antes apuntábamos y que tiene como sintético reflejo la existencia de una administración útil y servicial, no exenta de problemas y aspectos mejorables, pero sin duda útil e imprescindible para la atención y desarrollo de nuestro modelo de Estado.

\section{Credibilidad y confianza ciudadana: especial atención al control de la gestión pública}

En segundo lugar, debemos considerar la imprescindible y necesaria existencia de un vínculo de confianza entre la administración y los ciudadanos. Cuestión que, por su capital importancia, entendemos que debe ser un objeto de un tratamiento 
más extenso. Precisamente, la comentada utilidad de la administración y su real percepción incide de manera determinante en la existencia de la referida confianza y consecuente legitimidad de la actuación pública.

Por ello, es necesario, por un lado, esforzarse en desarrollar un modelo robusto y mejorado de gestión pública que, con resultados positivos apreciables por los ciudadanos, se pueda evitar la existencia de situaciones que se puedan filtrar discursos u opiniones irracionales o pseudoideológicas de nula aportación, y de muy negativo efecto en un clima de confianza.

Ahora bien, aunque es importante la idea de buena gestión (en definitiva de buena administración), no es menos importante el fuerte vínculo existente entre el control de la gestión y la confianza de los ciudadanos. Y es que, sin entrar en demasiados detalles, se puede afirmar que existe un notable déficit de credibilidad de los ciudadanos en sus instituciones y, por tanto, la legitimación de estas se encuentra muy mermada.

Este déficit de credibilidad $\mathrm{y}$, como decimos, de legitimidad, resulta democráticamente insano, pues es imposible de sostener a largo plazo. Tal vez a corto plazo, incluso a medio plazo.

Y resulta muy significativo que, en general, podemos hablar de esta patología en prácticamente cualquier lugar del mundo y ante cualquier opción política que tenga responsabilidades de gobierno. Patología generalizada hasta tal punto que podría hablarse de una auténtica pandemia de falta de legitimidad y esta última, más que un problema en sí mismo, se puede considerar una manifestación o negativo efecto de un auténtico problema de naturaleza sistémica, referido, en pocas palabras, al propio funcionamiento del sistema, a la pregunta que en cualquier momento un ciudadano puede llegar a hacerse: ¿cómo está funcionando el sistema? o, dicho de otra manera, ¿cómo se están gestionando sus asuntos?

Así las cosas, aunque se reconozca que la solución debería considerar la existencia de un espacio de mayor control, en el momento de abordar la solución al problema de falta de confianza, de credibilidad de los ciudadanos en la burocracia política que les representa, en la propia burocracia técnica de la administración y, por extensión, en las propias instituciones públicas, se puede identificar una etiología común.

Dicha etiología o causas se pueden clasificar en dos niveles: uno más visible y apreciable por el ciudadano a través de los múltiples casos de malas prácticas y corrupción pública. Y un segundo nivel que, no siendo tan apreciable, en buena medida ha posibilitado causas y efectos y que se refiere al abandono de los controles clásicos por el derecho público.

En todo caso sí debemos destacar que estas causas van a depender, sin duda, de las circunstancias sociales, políticas y económicas de cada país, cuestión sobre la que nos detendremos con más calma al abordar la limitada proyección de los procesos de reforma. No obstante, incluso teniendo presente esas circunstancias, podemos identificar una serie de factores comunes cuyo sumatorio han generado ese efecto 
negativo de falta de legitimidad o descreimiento social que conlleva un peligroso desafecto ciudadano hacia las instituciones sobre las que se sostiene el propio sistema democrático.

En efecto, por lo que se refiere al que hemos denominado como primer nivel de causas más apreciable al ciudadano, podemos identificar diversos factores bastante conocidos y renombrados:

- Los casos de corrupción, tan repetidos y variados en sus modalidades que ya, para algunos ciudadanos, parecen formar parte del propio sistema.

- La mala gestión, sobre todo en el sentido de gastar recursos sin rendimiento apreciable. En otras palabras, el gasto público de mala calidad.

- Los abusos de poder, asentados en privilegios o trato de beneficio que no aportan nada al desempeño de las funciones públicas de quien los disfruta y que ante los ciudadanos suponen, no ya un agravio comparativo, sino un factor inadmisible por cuanto es él quien lo está costeando.

Pero, como decíamos, estas causas tienen a su vez origen en un segundo nivel menos evidente pero perfectamente identificable vinculado a la metodología que se ha implementado y se implementa para la gestión de los asuntos públicos.

Nos referimos a la operación o tendencia de archivar el derecho público, el cual aunque en foros como este pudiera resultar baladí o reiterativo, es una herramienta fundamental para la defensa del interés general. Y una de las utilidades más importantes de ese derecho público, de esa eficaz herramienta para la defensa del interés general, es precisamente el control y la exigencia de responsabilidad.

Esta operación de archivo del derecho público se ha llevado a efecto a través de diferentes maneras, pero desde el punto de vista que aquí más nos interesa, el control de la gestión pública, una de las modalidades que más perjuicio ha podido causar (al interés general y, por tanto, a los ciudadanos) ha sido trasladar el centro de gravedad de la gestión pública a las técnicas y formas del sector privado.

Esto es, sin entrar en grandes disquisiciones jurídicas (que además ya han sido objeto de varios y profundos estudios en determinados ámbitos y momentos), el peso del derecho público y su contrastada significación en la defensa del interés general, han sido sustituidos por las reglas y metodología propia del sector privado.

Sustitución no simplemente técnica u operativa, ya que se ha llegado a asumir una auténtica filosofía de identificación de lo público con lo privado, mostrando todo lo privado como modelo o patrón de éxito cuando tal afirmación se muestra negada por una realidad de doble cara en la que, habiendo éxito y beneficio, también hay otro lado de fracaso y pérdida.

Y es que, esta operación no solo ha tenido un efecto interno o doméstico de preterición de las técnicas ordinarias de gestión y control de la actividad pública; podemos afirmar que llegó a generar en la conciencia colectiva, incluso al más alto nivel, una ilusión de éxito e infalibilidad de las formas de gestión privada que, como acabamos de indicar, es solo una de las caras de la moneda. El problema es que el fracaso de la forma privada de gestión en el sector público se paga con dinero de 
todos los ciudadanos. Escenario que, por lo que se refiere al control social y el de los ciudadanos, tuvo igualmente un efecto pernicioso al trasladar durante años de gran bonanza económica y vertiginosa creación de riqueza, una imagen distorsionada de la administración y de los gestores públicos, sobre todo al ser vistos como realidades no particularmente cercanas (salvo en periodo electoral, claro está), ni tampoco especialmente necesitados de un particular control. En definitiva, se abonó o se asentó la idea de que el dinero público, como recurso esencial para gestión pública, podía tratarse como si fuera dinero privado.

Recapitulando, a la vista de todo lo expuesto, el proceso de deslegitimación del sistema, ha tenido dos fases: en primer lugar, se ha implementado un modelo basado en la huida del Derecho Público o, en su caso, en la laxitud de su aplicación. En segundo lugar, como resultado de lo anterior, ante la ausencia o debilidad de los mecanismos de control y responsabilidad han ido apareciendo diversos casos y hechos de malas prácticas y corrupción que han nutrido la idea de la desconfianza, la falta de credibilidad en las instituciones y, por tanto, la deslegitimación de estas.

Ante este estado de las cosas, la reacción político-institucional es obvia y en un único sentido: recuperar el peso del derecho público, volver a situar el punto de gravedad en lo que realmente importa: la defensa del interés general. Algunos esfuerzos legislativos tanto a nivel nacional como internacional confirman esta reubicación ${ }^{2}$. Cambiar la operativa, la metodología es relativamente sencilla, por ejemplo, dando mayor importancia y virtualidad (a todos los efectos) a los órganos de control internos y externos, o recuperando normativas más estrictas y rigurosas. Pero, como señalaremos (Nota 8), la legitimación de un sistema no se alcanza exclusivamente con un cambio de operativa o de reglas de actuación a golpe de modificación normativa, pensando que la labor legislativa, por sí misma, tiene un "efecto panacea".

El factor social de la legitimidad de las instituciones no se regenera tan rápidamente como a veces se piensa, o nos gustaría, con un simple cambio normativo por muy atinado que este pueda resultar. Los ciudadanos no han reaccionado bien (o lo hacen como se comportaría cualquier ser humano) ante un escenario nada favorable: por un lado, los ya citados casos de malas prácticas y de corrupción. Por otro lado, la pérdida de muchos hitos del Estado del bienestar como consecuencia de una crisis que tiene entre sus causas una incorrecta gestión de recursos públicos y los propios casos de malas prácticas y de corrupción.

$\mathrm{Y}$ en su conjunto, el sumatorio de ese doble escenario, el resultado, es la pérdida

2 En España, sin perjuicio de las iniciativas legislativas adoptadas a nivel estatal, resultan muy destacables la labor legislativa desarrollada por varias comunidades autónomas, como Navarra y Extremadura. Desde su muy acertada y renombrada "Ley de improbidad administrativa" hasta la más reciente "Ley de Responsabilidad de las personas jurídicas por actos lesivos contra la Administración Pública Nacional y Extranjera". Ley 12.846, que ha instaurado una novedosa normativa anticorrupción, incorporando sanciones civiles y administrativas, contra personas jurídicas del sector privado, tanto locales como extranjeras. 
de grandes cantidades de dinero público, y la necesidad de reponerlo para hacer frente a necesidades y servicios básicos a través de la principal fórmula: la fiscalidad, con lo que ya tenemos el círculo cerrado en el que ha quedado atrapado el ciudadano.

Estos ciudadanos atrapados en la situación creada, enfadados, desconfiados, descubren en este momento la democracia, su significado $\mathrm{y}$, sobre todo, los postulados de transparencia y control en los que el modelo se asienta. Pero también descubren otros elementos del sistema democrático, absolutamente básicos, con los que no parecen estar de acuerdo, pareciéndoles incluso arbitrarios o abusivos; nos referimos a las titularidades activas que supraordenan a la administración y sus agentes, precisamente para la defensa del interés general. Y es que, la realidad les muestra que el empleo de estas titularidades, de esas potestades públicas de orden superior, su uso, abuso y abandono cuando ha interesado hacerlo (huida del derecho público) ha tenido como consecuencia los pésimos resultados ya expuestos y no tienen un alto nivel de confianza en quienes han de emplearlas.

La pregunta es inmediata, ¿cómo se obtiene de nuevo la confianza en las instituciones? Una primera idea ya la hemos expuesto: recuperar el valor del derecho público, procediendo a dotar de significación e importancia a los mecanismos de control y responsabilidad, de tal forma que supongan un tamiz para que los gestores públicos sean ejemplares por su virtud y su competencia. Ejemplaridad como primera instancia para que socialmente se genere mayor confianza y, por tanto, legitimidad. Las conductas ejemplares son cercanas y reconocibles para los ciudadanos. Ningún Gobierno puede considerarse legítimo si sus ciudadanos no confían en sus instituciones. Pero el problema no estaría resuelto si el esfuerzo no se extiende a la propia sociedad, a la base sobre la que se construye el sistema. No puede olvidarse que la burocracia política; las autoridades administrativas, los empleados públicos que proceden de esa sociedad, pueden ser los mismos ciudadanos que ahora protestan y reivindican una mejor gobernanza.

En este sentido, los ciudadanos, a través de la educación, la información y el conocimiento no deben olvidar que su capacidad de información y control es intrínseca al propio sistema democrático. Simplemente, los ciudadanos no deberían olvidar postulados fundamentales: el imperio de la Ley es una exigencia básica que beneficia a todos, incluso cuando particularmente no nos favorece.

Que no basta con el voluntarismo político o las buenas intenciones, que la técnica y el control a través del imperio de la Ley es garantía de objetividad y atención preferente al interés general. Que la transparencia como concepto síntesis para identificar el acceso a la información y el conocimiento de los asuntos públicos, no puede ser coyuntural o una simple moda.

Que la exigencia a los gestores públicos por los intereses de todos, el control de los asuntos que a todos nos afectan, es manifestación inequívoca de madurez cívica y, por tanto, de fortaleza democrática. Que la primicial y más importante manera de controlar y exigir es elegir (derecho de sufragio pasivo) responsablemente a quienes pueden proporcionar decisiones sensatas y adecuadas al interés general. En 
síntesis, cada ciudadano tiene su responsabilidad y tiene que proceder de acuerdo a ella.

El control de la actividad pública no puede ser una mera función administrativa o una simple técnica, ya que siendo muy importante la técnica o la regulación de la función administrativa, tiene que contemplar y ser contemplado desde una perspectiva social reflejada en valores y principios que, comúnmente reconocidos incluso por su consagración constitucional, generen confianza, credibilidad y, por tanto, solidez democrática.

\section{Administración y fortaleza democrática}

En tercer lugar, sin perjuicio de la actividad propia del resto de poderes, es necesario reconocer el determinante papel de la Administración y su actividad en el rendimiento y fortaleza de las bases constitucionales del Estado y, por tanto, del propio sistema democrático.

El buen funcionamiento de la administración, su utilidad a los ojos de los ciudadanos es la auténtica dignidad institucional de la estructura administrativa con un efecto secuencial: genera, como ya hemos visto, confianza, legitimidad, y, además, músculo democrático.

La relativa sensación de desgobierno que en la actualidad puede tener el ciudadano afecta a la propia consideración de la administración respecto a su utilidad y este no es un problema encapsulable o localizado en este ámbito del poder ejecutivo, tiene un fatal efecto sobre el conjunto del sistema democrático. Pero desbrozando estos planteamientos, que como bien sabemos, tienen una parte de veracidad insoslayable, no se puede negar que las administraciones siguen funcionando, siguen siendo útiles y únicas en la respuesta a las necesidades de los ciudadanos siendo su estructura la que, repetimos desde otro punto de vista, sostiene en gran medida el sistema democrático en el que el ciudadano ejerce sus derechos y libertades.

\section{Limitada proyección de los procesos de reforma}

En cuarto y último lugar, constatar que no existen fórmulas magistrales o no es posible hablar de modelos replicables. Cada proceso de cambio y mejora dependerá y estará modulado por las circunstancias políticas, sociales y, sobre todo, económicas de cada organización pública y de cada momento. Consideración que pone en primer orden de prioridad la necesidad de permanente adaptación a las nuevas realidades, echando por tierra el mito del inmovilismo administrativo.

En esta línea y en mayor consideración, resulta procedente recordar la opinión de la premio nobel de economía Elinor Ostrom, quien al analizar los fracasos institucionales y las posibilidades de autoorganización advierte sobre las cautelas que deben tenerse con el empleo de modelos abstractos que tratan de 
imponer panaceas institucionales universales para resolver problemas a pequeña escala, pero no por ello menos complejos, inciertos y difíciles. La aclaración de que el mundo es más complejo de lo que muestran estos modelos es obvia, y no es útil en sí misma (Ostrom, 2000, p. 291).

\section{La materialización de la mejora y el cambio}

A la vista de las consideraciones expuestas en el apartado anterior, puede apreciarse que, con carácter general, se dispone de una base teórica muy apreciable y evolucionada, junto con una notable experiencia para consolidar la idea de reformar la administración; base teórica y destreza que se nutre del tiempo transcurrido y los proyectos, programas y actuaciones relacionadas con la idea de mejora y cambio de la administración.

Es más, podemos decir que en el ámbito jurídico-social y, particularmente en el sector público, no ha habido históricamente un esfuerzo igual que el realizado por alcanzar el objetivo de una administración que, adjetivada de diferentes maneras (eficaz, eficiente, ágil, transparente, vicarial, cercana, entre otras), obtenga una unánime y positiva valoración por parte de los ciudadanos y los propios servidores públicos.

Sin embargo, pese al indudable y esforzado afán de cambio y mejora como se ha podido apuntar hace un momento, no se ha llegado a alcanzar un resultado óptimo y generalizado. Siendo benevolentes en la valoración, en los mejores tiempos del cambio de las administraciones, muchos y grandilocuentes proyectos de reforma administrativa solo han generado autocomplacencia y cuidadas, pero costosas presentaciones. La ausencia de resultados que la situación de crisis padece se ha mostrado de manera muy cruda, al vincular los defectos y máculas no corregidas de las distintas administraciones con parte de la etiología de la propia situación dificultosa. Lo anterior si no se evidencia en su origen, sí en su agravamiento o incapacidad de amortiguar sus negativos efectos.

Evidentemente no vamos a entrar en el examen del acierto o no de este tipo de planteamientos causalistas, pero sí conviene posicionarnos en este entorno de crisis económica y, para muchos, también institucional, para aprovecharlo como un espacio de revisión y oportunidades. Y la mejor conformidad, vista la ya apuntada base teórica y experiencia acumulada, es aprovechar el conocimiento que se ofrece desde el error y el fracaso, para que con inteligencia no se vuelvan a cometer errores o proceder a subsanar deficiencias inaceptables, tanto en tiempos de crisis como en periodos de mayor normalidad económica e institucional. Y ello con la finalidad última de generar ante los ciudadanos una visión de esperanza $\mathrm{y}$, por tanto, de un futuro mejor.

En efecto, los procesos legislativos desarrollados tanto a nivel nacional como autonómico, y las actuaciones llevadas a cabo de manera concreta por la práctica 
totalidad de las administraciones públicas, confirman la afirmación antes apuntada de proceder con inteligencia, tratando de recuperar el tiempo perdido con normas y medidas que hace tan solo un par de años pocos se habrían atrevido a proponer y mucho menos a ejecutar. Se trata, como decimos, de recuperar el tiempo perdido, pero también de tener un comportamiento más ejemplar ante el ciudadano, basado en la eliminación de cargas innecesarias susceptibles de liberar recursos económicos que puedan ser destinados a servicios más esenciales y cercanos a los propios ciudadanos.

$\mathrm{Si}$ analizamos los procesos de reforma $\mathrm{y}$, por tanto, de mejora de la administración, podemos sintetizar una serie de elementos conformadores de una matriz o denominador común que en su conjunto conforman una receta muy simple que, salvando el modelo de administración expresado en nuestra Constitución y por tomar un hito cronológico cercano, ya era conocida desde que el legislador elaboró la Ley de Régimen Jurídico de las Administraciones Públicas y el Procedimiento Administrativo Común, cuyos preceptos tan acertados, por ejemplo, en materia de creación de órganos, cooperación entre administraciones o ejercicio de competencias podemos decir que fueron congelados por una glacial tormenta de confianza en las formas de gestión privada, la indiferencia y desatención de gestores públicos y ciudadanos respecto a la importancia del derecho público, y una situación económica favorable que permitía hacer de todo y sin preguntas a corto plazo, pero que resultaba, como muy bien y dolorosamente sabemos, insostenible a largo plazo.

En la actualidad, simplemente se están descongelando aquellas viejas reglas, paliándose en algunos casos la necrosis del sistema al recuperar principios y valores que vuelven a ser considerados importantes, a saber:

- Respeto al principio de competencia (titularidad activa) considerando, por extensión, su carácter irrenunciable.

- Deber de coordinación en la misma administración y de interacción cooperativa entre administraciones.

- Sujeción a los límites y condicionantes que modulan el ejercicio de la potestad organizatoria.

- Vigencia de los principios de mérito y capacidad en el acceso a cargos y funciones públicas de carácter ejecutivo.

- Mayor respeto a los órganos de control interno y externo como instrumentos de prevención y, por tanto, de disuasión cuando no de reacción si fuera necesario.

- Proximidad y servicio real al ciudadano a través de un comportamiento y una estética marcada por la sencillez y la simplicidad, permitiendo que el ciudadano conozca y entienda. Y al conocer y entender, se incremente su sensación de seguridad y confianza en la administración.

No se trata de trivializar instituciones y procedimientos, sin duda, complejos, pero frente al ciudadano medio hay que realizar planteamientos sencillos, soslayando 
obstáculos u oscuridades con los que la sensación es de inseguridad y desconfianza. Aspectos que nos definen el modelo de administración que se desea y que, en definitiva, es coincidente, con los perfiles constitucionales que ya se marcaron hace treinta y cinco años, posteriormente desarrollados, como decíamos líneas atrás, infelizmente preteridos y ahora recuperados con la clara intención de aseguramiento y definitiva consolidación.

Como se ha podido apuntar ya, se trata de una recuperación del derecho público que se diferencia de anteriores y fallidos proyectos en que el planteamiento se hace sin complejos, con la valentía que proporciona la necesidad, y sabiendo que la ciudadanía no admitiría que, al final, solo se tratará de una mera declaración de buenas intenciones tal y como ha sucedido en otras ocasiones. En este momento, diferentes y relevantes hechos legislativos y actos administrativos, avalan de manera objetiva y contundente que la reforma de la administración se está llevando a efecto. Reforma de la administración generadora de competitividad institucional ${ }^{3} \mathrm{y}$, en consideración, posibilitadora de crecimiento y fortaleza del Estado del bienestar; y es que las políticas de crecimiento económico han de ser paralelas y/o conmutativas a las políticas de crecimiento institucional.

\section{TRANSPARENCIA: DESDE EL CONOCIMIENTO A LA REGENERACIÓN DEMOCRÁTICA}

Las actuaciones en materia de transparencia de la administración se han convertido en una preocupación global, tal y como por ejemplo se ha puesto de manifiesto en la Conferencia Internacional Open Government Partnership celebrada en Brasilia durante los días 17 y 18 de abril de 2012 y que ha tenido como resultado una nueva declaración que se une a las ya existentes hasta la fecha, la denominada: Declaración Internacional de Gobierno Abierto.

Sin entrar en grandes disquisiciones sobre el concepto de transparencia y su tratamiento a nivel normativo y doctrinal, entendemos que una de las visiones más acertadas que se puede tener de la transparencia es su consideración desde una perspectiva social. Esto es, considerar que la transparencia no puede ser únicamente una función administrativa o un mero comportamiento solicitado por una norma. $\mathrm{Si}$ entendemos que la información jurídica y económicamente relevante, el divulgado conocimiento de las instituciones y organizaciones públicas, sus actos y personal tiene como principal (y podíamos decir, único) destinatario al ciudadano, supone

3 No es posible obviar que en el actual marco jurídico institucional, los Estados compiten entre sí y por nuestra configuración territorial, las comunidades autónomas son competitivas entre sí. No es posible En otras palabras, las instituciones públicas son altamente competitivas teniendo en su organización y estructura administrativa como prestadora de los servicios que le son propios, un fundamental aspecto que diferencia y determina su imagen y, lo que es más importante, capacidad de generar confianza o, en su caso, atracción. 
que dicha información, como efecto de la denominada transparencia ha de facilitar la participación y el control, coadyuvando entonces a generar una mejor gestión pública.

Bien es cierto que el acceso a la información ya estaba regulado en la Ley de Procedimiento Común (artículos 35 y 37), además de una tendencia anudada a las mayores exigencias ciudadanas por la materia, teniendo como resultado que a día de hoy existe un volumen importante de información puesto a disposición de los ciudadanos a través de los canales oficiales de la práctica totalidad de las administraciones públicas. La novedad se identifica en varios aspectos:

- Ampliación del ámbito objetivo del acceso.

- Acceso a través de formatos que permitan la reutilizar esa información.

- Establecimiento de modalidades de información que permitan cualquier ciudadano medio un conocimiento adecuado y por tanto valorable por el mismo de la gestión pública.

La información no puede ser distante, alejada al ciudadano.

- Posibilidad de exigir responsabilidad en caso de incumplimientos sobre la materia.

- Creación o disposición de órganos ya existentes para velar por el cumplimiento de los nuevos parámetros normativos reguladores de la transparencia administrativa.

Aspectos que nos sitúan ante un planteamiento concreto en materia de transparencia y lo que es más importante, los efectos de esta para establecer un control posible, real alejado de los teóricos planteamientos de una transparencia ideal $\mathrm{y}$, consecuentemente, un control ideal que pese a su bondad y común aceptación la experiencia nos muestra que resulta complejo su paso de planteamiento teórico a la realidad.

\section{RESPONSABILIDAD: DESDE EL OLVIDO A LA EJEMPLARIDAD}

\section{Planteamientos generales}

Tratar de plantear algo medianamente novedoso en este ámbito resulta una tarea complicada a partir de lo que la doctrina más autoriza y nosotros mismos hemos podido escribir al respecto. Y ello sin perjuicio de la situación cercana al agotamiento que se puede percibir en interesados y operadores. No obstante, más allá del endurecimiento de las medidas de control y respuesta sancionadora o, en su caso, penal, la realidad nos continua sorprendiendo con conductas que evidencian un escaso apego al ejercicio de la función pública en términos de adecuación jurídica y, podría decirse, en parámetros de corrección política. Y la sorpresa no es tanto por los hechos en sí (que en ocasiones asombran) como por la aparente normalidad con la que algunos servidores públicos realizan determinados comportamientos que, ya en su origen, cualquier persona con un conocimiento jurídico medio o incluso sin 
conocimiento alguno, pero con la normal sensatez de un correcto ciudadano (un buen padre de familia que opta por el servicio público), inmediatamente trataría de evitar ${ }^{4}$.

$4 \quad$ Un breve vistazo a la jurisprudencia española más reciente nos muestra significados ejemplos de ello. Así, la STSJ Valencia de 19 de febrero de 2013 (La Ley 486/2013) por la que se condena a un alcalde por prevaricación debido a la arbitraria adjudicación de un concurso público para la recogida de residuos. La autoridad municipal manipuló el proceso de adjudicación mediante un complejo sistema para conseguir adjudicar el contrato a una concreta empresa. Para la Sala se llevó a cabo una actuación arbitraria y lesiva para el interés público, mintiendo con una falsa consulta a tres despachos de consultoría externa para dotar al proceso de una apariencia de legalidad, pues ante las opiniones discrepantes de los técnicos municipales anunció por primera vez en la historia del Ayuntamiento, que iba a recurrir al asesoramiento externo mediante la petición de informes a tres despachos de abogados (mínimo exigido por la ley de contratación pública) pero en realidad solo consultó con un despacho, vinculado laboralmente con la empresa que consiguió la adjudicación. La SAP Murcia de 12 de enero, 2012 (La Ley 1604/2012), refleja la prevaricación cometida por la Presidenta y vocales de Junta Municipal, que de modo arbitrario votaron, en Pleno Ordinario, contra la concesión de subvenciones municipales a una asociación de vecinos que reunía todos los requisitos para ello. El único motivo alegado fueron las continuas críticas a la gestión de la Junta Municipal expresadas en la revista que la asociación editaba periódicamente. Para la Audiencia, las autoridades actuaron con plena conciencia de que su resolución era decidida al margen del ordenamiento jurídico, anteponiendo su voluntad a cualquier otra consideración, ocasionando un resultado discriminatorio y materialmente injusto.

Por su parte, la SAP Islas Baleares de 19 de marzo, 2012 (La Ley 22400/2012) nos muestra con crudeza la condena a 6 años de prisión a un Presidente de Comunidad Autónoma por delitos de fraude a la administración, falsedad documental, prevaricación, malversación y tráfico de influencias. Sentencia que pone de manifiesto, en primer lugar, el concierto entre funcionarios y particulares en el ámbito de la contratación administrativa para defraudar a la Comunidad Autónoma, facilitando información previa sobre futuro concurso a publicar y proporcionando propuestas adicionales para mejorar la oferta frente a posibles licitadores. En segundo lugar, el pago de comisiones a la sociedad interpuesta como contraprestación por posibilitar el artificio administrativo. En tercer lugar, la petición de apoyo económico para proyecto empresarial con prevalimiento o influencia sobre la autoridad, consiguiendo una resolución beneficiosa económicamente. En cuarto lugar, la concesión de subvenciones, desviando fondos de su importe para el pago de prestaciones y servicios de otra actividad. Y en quinto lugar, falsedad documental.

En una línea similar puede apuntarse la SAP Sevilla de 18 de marzo, 2011 (La Ley 165464/2011) en la que se pone de manifiesto la falsificación por un concejal de las facturas del móvil oficial del Alcalde al objeto de encubrir el delito de malversación cometido por este al destinarlo exclusivamente a llamadas particulares.

Significativa es también, con independencia del fondo procesal y el fallo de la misma, la STS 15 de marzo, 2012 (Diario La Ley, No. 7845, 25 abril 2012) en la que se pone de manifiesto la realización de contratos entre el Ayuntamiento y diversas empresas participadas y gerenciadas por un concejal del mismo con el beneplácito del alcalde, y todo ello, con la mediación de un acuerdo político de "gobernabilidad".

También puede recordarse la SJPenal de 17 de febrero de 2012 (La Ley 8653/2012) relativa a la prevaricación de un Alcalde, en comisión por omisión, al permitir las obras de rehabilitación de un hotel sin licencia y no incoar el preceptivo expediente de infracción urbanística por cuanto hizo caso omiso a las denuncias presentadas por un funcionario municipal, sin adoptar ningún tipo de resolución administrativa encaminada a poner fin al estado de ilegalidad.

Finalmente, por el perfil profesional afectado, llama la atención la STS de 28 de febrero, 2012 (La Ley 15840/2012), por la que se confirma la sanción impuesta a un notario que llegó a autorizar más de cien escrituras y actas diarias por ser imposible que estuviera presente en el otorgamiento de todas ellas.

A mayor abundamiento, en un campo en el que se mezcla la gestión privada con la iniciativa 
Hoy, una de las malas prácticas como la corrupción es una de las grandes preocupaciones de los ciudadanos cuyo efecto es devastador. Lo es para los propios ciudadanos que pierden la confianza en las instituciones que les representan y que se encargan de gestionar sus intereses; pero también lo es para los propios gestores públicos que desempeñan sus funciones con corrección y ejemplaridad, ya que la situación, el contexto generalizado produce en todos ellos un lacado de desconfianza y ausencia de todo aprecio a su trabajo.

El sumatorio de estos dos elementos impacta sobre la propia democracia, la debilita, le genera una grave patología de raquitismo, retrocediendo años ${ }^{5}$. Y buena parte del problema trae causa en la idea de responsabilidad por la gestión, cuestión que no solo tiene un enfoque estrictamente jurídico.

La responsabilidad por la gestión tiene, indudablemente, que encuadrarse en un orden jurídico que discipline comportamientos y reaccione en caso de incumplimiento. Si así fuera, los ciudadanos, cualquier interesado en la materia no estaría tan preocupado ya que, sin perjuicio de modulaciones, la realidad nos muestra que el Estado de Derecho está respondiendo adecuadamente, al menos, en el extenso uso de uno de sus instrumentos que es el derecho penal.

Desde nuestro punto de vista, la cuestión medular es que, como veremos más delante de manera más precisa, la conciencia de responsabilidad en la gestión de los asuntos públicos no se adquiere a fuerza de norma jurídica o por una especie de epifanía jurídica que recibe el lector de un boletín o diario oficial. El derecho, ayuda, protocoliza, disuade pero no tiene plena capacidad para generar el fundamento ético, moral que todo ciudadano debe tener interiorizado y que marca su posición y conducta respecto al interés general.

\section{Responsabilidad y control: nuevas perspectivas}

Resulta innegable entender que la idea de responsabilidad se anuda necesariamente al control. En un planteamiento muy simple puede afirmarse que la ausencia de responsabilidad no es más que un efecto del mal funcionamiento de los sistemas de control.

y proceder público, no podemos desconocer por sus muy lamentables efectos el caso de las cajas de ahorro que sin conocerse a esta fecha sentencia alguna, ya son objeto de atención por parte, entre otros, de la Fiscalía General del Estado, quien ha ordenado depurar todas las responsabilidades penales derivadas de la gestión de varias cajas de ahorro afectando a indemnizaciones de directivos, delitos de estafa, apropiación indebida, falsedad documental o fraude fiscal. En este sentido, se ha solicitado al Banco de España la más completa colaboración y una mayor agilidad a la hora de entregarle la documentación solicitada (Diario La Ley, No. 7863, Sección "Hoy es Noticia", 21 Mayo 2012). Noticia que vuelve a mostrarnos un panorama poco ejemplificador y alejado de lo que cualquier ciudadano medio podría entender como gestión diligente o adecuada.

5 La comparación de la situación de España con la vivida en Italia con el denominado caso "tangentópolis" resultando injusta, no puede ser más elocuente. Recientemente se ha vivido en Brasil un caso de corrupción institucionalizada, el denominado caso "mensalão" que igualmente se ha tratado de comparar con la situación española. 
Y planteada la cuestión relativa a los sistemas de control, no resulta muy difícil admitir que, al menos, hasta la fecha y de manera general, los sistemas de control han tenido unos resultados bastante pobres por no decir deficientes. En consecuencia, en línea con lo antedicho al citar los conceptos de transparencia ideal y transparencia real, resulta necesario empezar a hablar de un control posible adecuado que debe comenzar desde la generalidad hasta descender al caso más concreto. Es decir, podemos plantear la idea de un principio de control que ha de vertebrar vertical y horizontalmente la organización pública.

A su vez, el replanteamiento del principio de control debe conducirnos a reconsiderar el objeto de dicha inspección, que comenzando al más alto nivel (para continuar, como decimos, en un descenso a situaciones más particulares) nos sitúa ante la perspectiva de vigilar las políticas públicas. Estas últimas deben ser entendidas como auténticas líneas de actuación general con capacidad de marcar el desarrollo de una nación o, en su caso, una región a todos los niveles y, lo que es más importante, a largo plazo. No es posible y entendemos que ha sido un error, identificar las políticas públicas con programas gubernamentales cuya limitada proyección temporal y carácter, en no pocas ocasiones, coyuntural, excluye su conceptuación como auténtica política pública de Estado, de nación con visión, repetimos, a largo plazo, generacional. Las necesidades a corto plazo son importantes pero las mejores soluciones se plantean a largo plazo.

No es sencillo plantear el establecimiento de políticas públicas en el sentido apuntado. Pero proponer principios que cumplan con pautas constitucionales ajenas a cualquier opción partidista, un denominador común a cualquier programa político, debe ser considerado como una manifestación inequívoca de madurez social y, particularmente, de los representantes ciudadanos.

Solo desde la prioridad constitucional de objetivos es posible construir la anunciada idea de control y responsabilidad en la gestión, evitando con carácter apriorístico, los peores efectos de la mala gestión pública plasmados en la absorción de recursos públicos sin rendimiento o la ejecución de macroproyectos sin rendimiento social alguno ${ }^{6}$. Y es que nos hemos acostumbrado al control de bajo nivel por su concreción, por ejemplo, la ya comentada acción penal contra un cargo público concreto que, siendo importante, no colma las necesidades de control que requiere la gestión pública. No es por ahí, al menos de manera exclusiva, por donde deben orientarse los esfuerzos en orden a fortalecer el principio de control y, por tanto, la exigencia de responsabilidad: este compromiso por la gestión debe partir desde el mismo momento de plantear las grandes políticas públicas que determinan la mayor o menor atención a las necesidades y expectativas de los ciudadanos mediante el

6 Baste pensar en el elocuente ejemplo de los aeropuertos construidos u otras infraestructuras públicas sin que hayan llegado a cumplir ninguna de las finalidades que pudieran preverse en su inicial planteamiento, siendo consideradas por los ciudadanos y los propios gestores como inútiles. 
óptimo empleo de recursos públicos y la constitución de estructuras administrativas prestadoras de servicios de calidad y sostenibles a largo plazo.

En síntesis, a partir de esta noción de política pública que excluye la tradicional visión de meras políticas de gobierno, es posible empezar a dar real y adecuado cumplimiento al principio de control y, en esta medida, disponer de indicadores objetivos para la exigencia de responsabilidad a través de las prioridades constitucionales ajenas a todo conflicto. En este sentido, no es por ejemplo baladí la incorporación del principio de estabilidad presupuestaria al artículo 135 de la Constitución y general vinculación de los gestores públicos a este principio, cuyo incumplimiento tiene que tener un claro reproche en términos de responsabilidad. Y es que, cabría preguntarse, ¿quién tiene más responsabilidad? El que pone en peligro servicios públicos esenciales para el mantenimiento del Estado del bienestar por llevar a cabo una gestión pública asentada en un endeudamiento público sin mayor rendimiento social que la existencia de grandes infraestructuras sin utilidad, o el que verifica un tipo infractor de cohecho o prevaricación, ¿quién menoscaba de manera más grave el bien jurídico, interés general, que representa la administración? Evidentemente en este segundo caso existe responsabilidad y en el planteamiento que hemos hecho debe ser depurada a través de la vía penal, o en otro caso, por la vía administrativa, pero es indudable que no puede quedar sin respuesta aquel que pone en peligro al propio sistema a través de una gestión poco diligente, alejada de cualquier prudencia y mínima pericia técnica, no generadora de ningún tipo de beneficio neto social, más bien al contrario, generador de una carga a heredar por generaciones futuras. Y es este uno de los más graves problemas de legitimidad y credibilidad ante los ciudadanos que observan con pasmo cómo gestores públicos adoptan decisiones cuestionables, incluso arbitristas, al no existir tacha de legalidad ordinaria; abandonan su puesto sin responder de su mala gestión y las fatales consecuencias de esta para el conjunto de los ciudadanos. Todo ello porque no existe control alguno en relación a las que hemos denominado grandes políticas, absteniéndose de cualquier tipo de parámetro que conduzca a su evaluación, más allá, claro, del propio sistema electoral. Sin duda, este estado de la cuestión debe ser superado, no limitarse a la tradicional vinculación positiva (hacer) o negativa (abstenerse de hacer) a la Constitución susceptible de tacha de inconstitucionalidad, debemos configurar esta como un auténtico parámetro de validez de la actuación administrativa mediante la destilación de ese prioritario denominador común de arranque constitucional conformador de esas grandes políticas públicas o, en otras palabras, posiblemente más cercanas, políticas de Estado que necesariamente han de tener resultados en favor de la sociedad susceptibles de renovar y, en su caso, reforzar la confianza de los ciudadanos. 


\section{CALIDAD DE LOS SERVICIOS: DESDE LA IDEA INALCANZABLE A LA REALIDAD MEDIANTE LA EVALUACIÓN}

En la actualidad, proyectar el tema de la calidad de los servicios supone realizar un planteamiento de doble frente. En primer lugar, partir de una posición realista desde la que se sea consciente de la necesidad de dotar a la sociedad de una estructura administrativa sostenible a largo plazo. Esto es, dando respuesta a las necesidades y expectativas del presente, no comprometer el bienestar de las generaciones futuras. Buen ejemplo de ello lo podemos encontrar en el cuestionamiento de la estructura administrativa generada para el desarrollo de los denominados derechos sociales, hoy objeto de cuestionamiento y revisión, hasta el punto de plantear, sencillamente, que muchos de estos derechos no funcionan por no poder ejecutarse correctamente ${ }^{7}$.

En segundo lugar, la recuperación de la técnica en la gestión como, de hecho, se está haciendo tal y como hemos podido anticipar al analizar las medidas que en estos momentos de grave y profunda crisis se están adoptando. Técnica que, como hemos podido apuntar al comentar la función social del control, debe estar informada de los valores y principios que, incluso con un arranque constitucional, definen el marco más trascendente pero perfectamente reconocible por los ciudadanos en el que ha de desarrollarse la gestión pública ${ }^{8}$.

Calidad de los servicios que supone el efecto o manifestación inequívoca de un modelo de administración alineado con los parámetros apuntados líneas atrás que, desde el impacto de la actual situación económica que se padece y a la que hemos hecho referencia en varias ocasiones, implica disponer de una administración que posibilita un espacio de creación de riqueza y, por tanto, de generación de empleo. $\mathrm{Y}$ es que, combinando las ideas expuestas de sostenibilidad a largo plazo y técnica en la gestión, debemos afirmar sin complejos que la administración por sí sola no es capaz de sostener el sistema. En otras palabras, la calidad de los servicios ha de posibilitar la existencia de un estado de bienestar social asentado en una economía lo suficientemente fuerte, sólida, que asegure un Estado social y democrático que no quede comprometido por la imposibilidad de alcanzar metas insostenibles a largo plazo o que, incluso, le puedan llegar a provocar su propio colapso.

La actuación pública debe marcar el camino de un sistema adecuado a la realidad social y económica ${ }^{9}$, teniendo como herramientas de ejecución y desarrollo unos

Baste pensar en la sanidad universal y gratuita con un catálogo de prestaciones muy amplio, o los derechos vinculados a la protección y ayuda a la dependencia.

8 Muy en la línea de lo que apuntaba Tocqueville respecto al universo moral que conforman las sociedades democráticas. Sobre esta cuestión puede consultarse el trabajo de Durán (2008, p. 4).

9 Sin entrar ni justificar un anacrónico intervencionismo de la administración en la actividad económica, la realidad nos muestra la necesidad de algún tipo de regulación que asegure el mejor y más adecuado espacio económico. Buen ejemplo de ello es lo acontecido en el sector financiero y lo que de manera generalizada se solicita a los poderes públicos en general y particularmente a la Administración. Sector financiero no ajeno a una constante polémica sobre la capacidad pública de su regulación y que sea como sea, siempre ha sido un referente de atención al hablar sobre la materia. 
servicios públicos de calidad. Adecuación a la realidad y servicios de calidad que vienen a ser el resultado de un buen funcionamiento de la administración como superior órgano gestor del Estado con base en una previa planificación asentada en principios que han de informar la actuación del conjunto de la burocracia política tan esenciales como el de continuidad de políticas públicas, al objeto de posibilitar el ciclo temporal necesario para cerrar el círculo virtuoso de la calidad a través del desarrollo, evaluación de resultados, corrección y consecuente perfección de la prestación del servicio.

Es claro que el elemento medular del que hemos denominado círculo de la calidad de los servicios es la evaluación, la cual podría, más bien debería, dar respuesta a las preguntas que siempre se plantean sobre la eficacia, eficiencia, resultado de la actividad administrativa a través de sus servicios y que, en la mayoría de las ocasiones, no reciben respuesta alguna o, a lo sumo, vagas o imprecisas contestaciones más teóricas o doctrinales que reales. Es más, evaluación de los servicios que implica un aspecto ya abordado en estas páginas como es el control de la gestión en el sector público y el cumplimiento de los objetivos políticos planteados ante los ciudadanos.

\section{REFERENCIAS}

Durán, J.F. (2008). Durkheim y Tocqueville: dos visiones sobre el papel de la religión en el mundo moderno. Nómadas. Revista Crítica de Ciencias Sociales y Jurídicas, 20.

Ostrom, E. (2000). El gobierno de los bienes comunes. La evolución de las instituciones de acción colectiva. México: UNAM-CRIM-FCE.

En este sentido, desde la perspectiva actual y el proceder público respecto al mercado financiero y propio comportamiento de este en la etiología de la crisis económica y su solución, resultan reveladoras las palabras que, en lo tocante a la "dictadura económica" de algunos operadores financieros, su Santidad Pio XI en 1931 recoge en la Carta Encíclica Cuadragésimo Año, sobre la restauración del orden social en perfecta conformidad con la Ley Evangélica: "Salta a los ojos de todos, en primer lugar, que en nuestros tiempos no solo se acumulan riquezas, sino que también se acumula una descomunal y tiránica potencia económica en manos de unos pocos, que la mayor parte de las veces no son dueños, sino solo custodios y administradores de una riqueza en depósito, que ellos manejan a su voluntad y arbitrio.

Dominio ejercido de la manera más tiránica por aquellos que, teniendo en sus manos el dinero y dominando sobre él, se apoderan también de las finanzas y señorean sobre el crédito, y por esta razón administran, diríase, la sangre de que vive toda la economía y tienen en sus manos así como el alma de la misma, de tal modo que nadie puede ni aun respirar contra su voluntad.

Esta acumulación de poder y de recursos, nota casi característica de la economía contemporánea, es el fruto natural de la limitada libertad de los competidores, de la que han sobrevivido solo los más poderosos, lo que con frecuencia es tanto como decir los más violentos y los más desprovistos de conciencia".

http://www.vatican.va/holy_father/pius_xi/encyclicals/documents/hf_pxi_enc_19310515 quadragesimo-anno_sp.html (parágrafos 105 a 107). 
